\title{
Characteristics of Stern Waves Generated by Ships of Simple Hull Form
}

\author{
(First Report) \\ by Yasuaki Doi*, Member Hisashi Kajitani**, Member \\ Hideaki Miyata**, Member Shozo Kuzumi**, Member
}

\begin{abstract}
Summary
The characteristics of stern-waves generated by a deep-drafted model of simple hull form are experimentally investigated. The results show that nonlinear waves exist in the neighborhood of the stern and these waves are essentially same with free surface shock waves around bows in many respects except for the interaction with linear bow-waves and boundary layer. This interaction makes the stern wave formation more complicated having movement of starting point and change of strength. A simple calculation shows that the movement of starting point and attenuation of the stern waves remarkably influence the linear wave system in the far-field.
\end{abstract}

\section{Introduction}

Two kinds of investigations for the improvement of the aft-part of hull forms have been undertaken at the Experimental Tank of the University of Tokyo. One is the improvement of propulsive efficiency by applying potential theories $^{1)}$, and the other is development of stern-endbulb $^{2)}$. Both methods developed have turned out successful for the improvement of practical hull forms.

However, it is noted through the course of investigations that wave making in the neighborhood of sterns is very complicated with peculiar appearance ${ }^{122) 335}$. The waves around sterns seem to interact with boundary layer and be similar to nonlinear waves called free surface shock waves (abbreviated as FSSW) around bows ${ }^{34) 10)}$. To further develop the method of hull form improvement stress must be focused on the nonlinear stern waves which cannot be interpreted by potential theories.

In this first report wave making in the neighborhood of a stern of a simple deep drafted model is investigated. In Chapter 3 formations of linear and nonlinear waves and their relation with wave resistance curve are described together with boundary layer calculation. An approximate

\footnotetext{
* Hiroshima University
}

** The University of Tokyo treatment of the calculation of linear waves is attempted in Chapter 4. The detailed characteristics of nonlinear waves and their interaction with boundary layer are experimentally investigated in Chapters 5 and 6, respectively.

\section{Model ship}

A wall-sided deep-drafted model of simple mathematical hull form S-103, which was previously used by Inui ${ }^{6)}$, is served for the present study. The principal dimensions are

$$
L \times B \times d=2.000 \mathrm{~m} \times 0.1788 \mathrm{~m} \times 0.500 \mathrm{~m}
$$

The source strength $m(x)$ is linearly distributed on the centerplane as follows so that the distribution of the source is equivalent to the hull form in double model flow.

$$
m(x)=-0.4 U x / l \quad-l<x<l
$$

where

$$
l=L / 2
$$

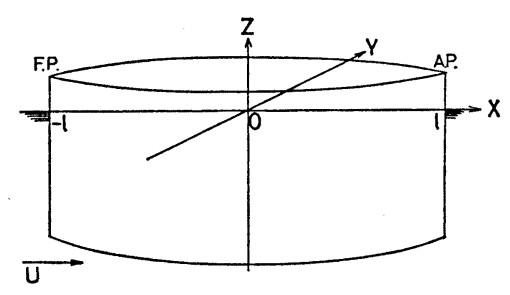

Fig. 1 Coordinate system 
$U$ is the speed of uniform stream and, as shown in Fig. $1,(x, y, z)$ are the Cartesian coordinates fixed in the ship.

The model is equipped with studs in a row at s.s. $9-1 / 2$ as a turbulent stimulator. They are $1.5 \mathrm{~mm}$ in height and with the spacing of $10 \mathrm{~mm}$.

\section{Characteristics of stern waves}

\subsection{Observation of stern waves}

The pictures in Fig. 2 show wave patterns around the full length of the model and those in the neighborhood of the stern. The former

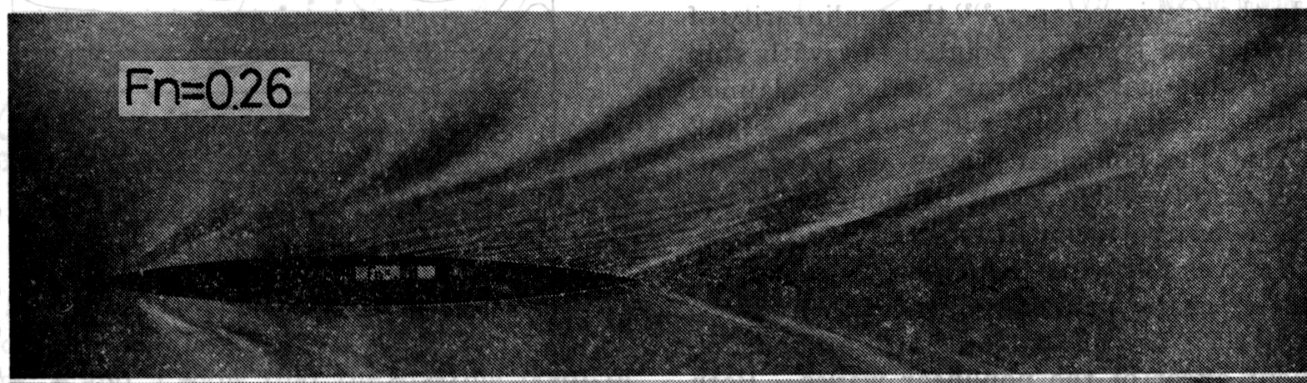

\section{$\mathrm{Fn}=0.27$}

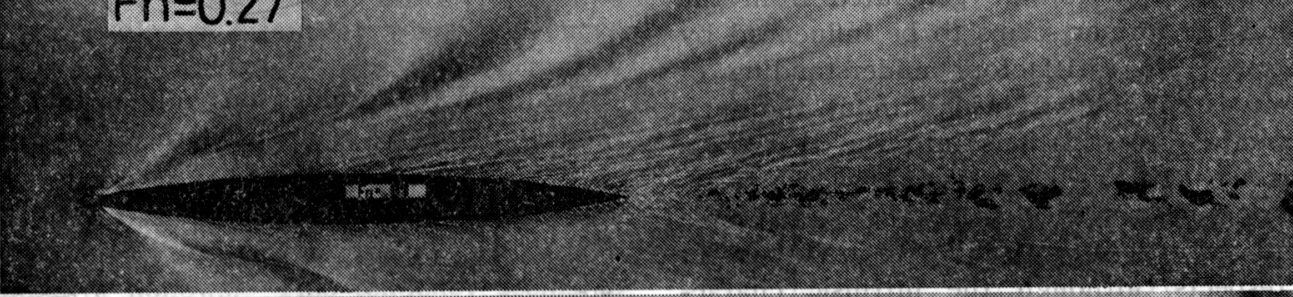

\section{$\mathrm{Fn}=0.28$}

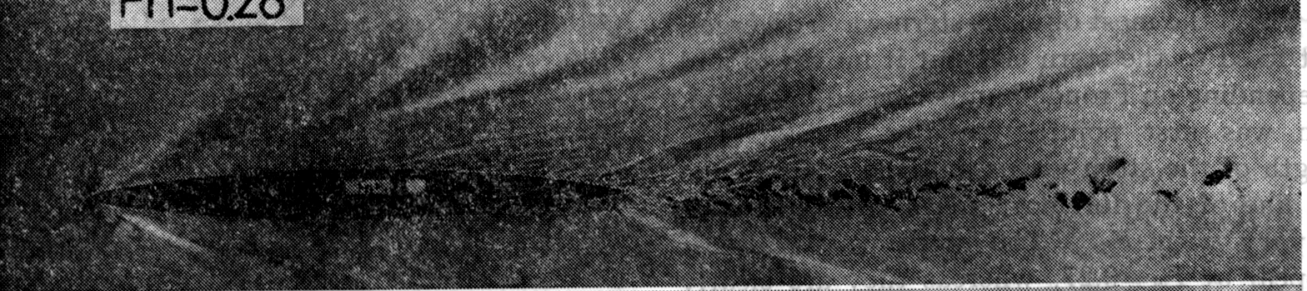

\section{$F n=0.30$}
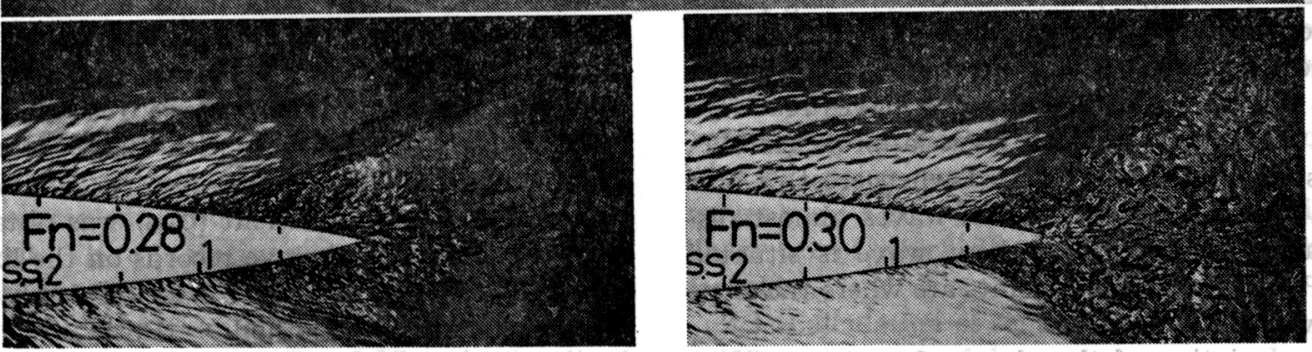

Fig. 2 Wave pattern pictures 
pictures are photographed with aluminum powder film on the water surface and the latter without it.

These pictures indicate that a kind of nonlinear wave occurs in the neighborhood of the stern showing lines of discontinuity at the wave front across which aluminum powder cannot smoothly pass. In the previous papers ${ }^{233}$ the nonlinearity of the waves from sterns is pointed out with ship models of practical hull forms, and it is claimed that these waves are FSSWs at the stern. Fig. 2 shows that the nonlinear waves are most conspicuous at $F_{n}=0.30$ among the four being accompanied by the turbulence of the free surface, and the waves following them seem to propagate dispersively in the manner of linear Kelvin waves, although they are shifted outward by the displacement effect of the nonlinear waves. Both linear dispersive waves (Kelvin waves) and nonlinear waves (FSSWs) are generated from the stern and the former seems to be influenced by the latter. No satisfactory understanding of stern waves can be attained without thorough experimental investigations into the two wave systems.

The nonlinear waves from the stern have different features from FSSWs around bow in some respects, although the intrinsic characteristics are the same as will be described in Chapter 5. The starting point of stern waves, from which the most obvious stern wave diverges, is not fixed to the stern end but it moves to and fro depending on Froude number based on $L\left(F_{n}\right)$, which was first pointed out by Inui. ${ }^{7)}$ The strength of the stern waves and the angle of the front line of the foremost stern wave to $x$-axis also vary with $F_{n}$. The stern waves almost disappear at $F_{n}=0.27$, whereas they are clearly observed both at $F_{n}=0.26$ and 0.28 . Besides, small increase of advance speed produces outstanding stern waves at $F_{n}=0.30$ which are greater than bow waves and they are weakened again at $F_{n}=0.37$.

3.2 Wave formation and wave resistance curve

The periodical change of the stern waves is related with the phase of bow waves. The wave profiles on the starboard side of the hull surface are shown in Fig. 3, in which the starting points of stern waves are marked by $\downarrow$. The wave profiles lacking with $\downarrow$ do not have obvious stern waves. The starting point moves rearward when a trough of bow wave approaches AP, and suddenly moves forward when crest of bow wave approaches AP. Stern waves almost disappear when a crest of bow wave is situated at AP. All the starting points are where the slope of bow wave is positive.

The variation of the observed position of the

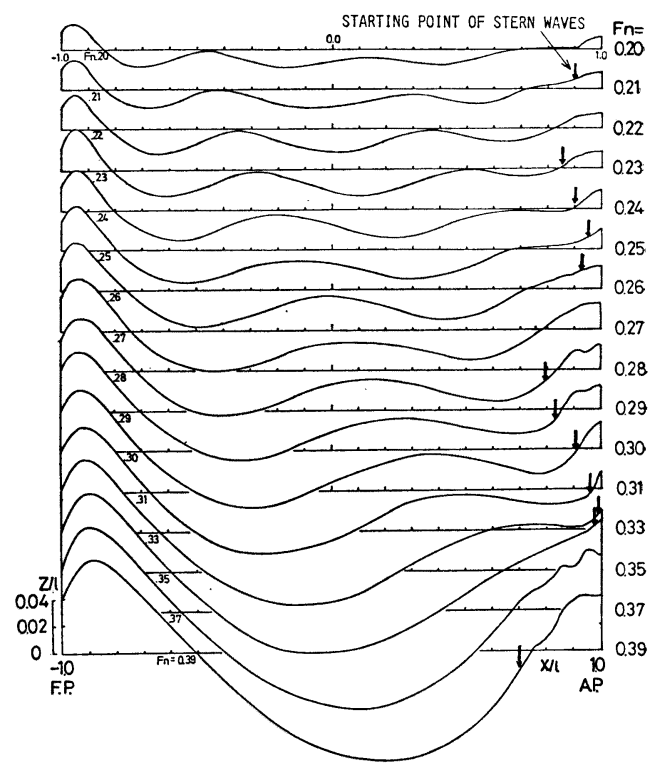

Fig. 3 Wave profiles on hull surface and starting points of stern waves

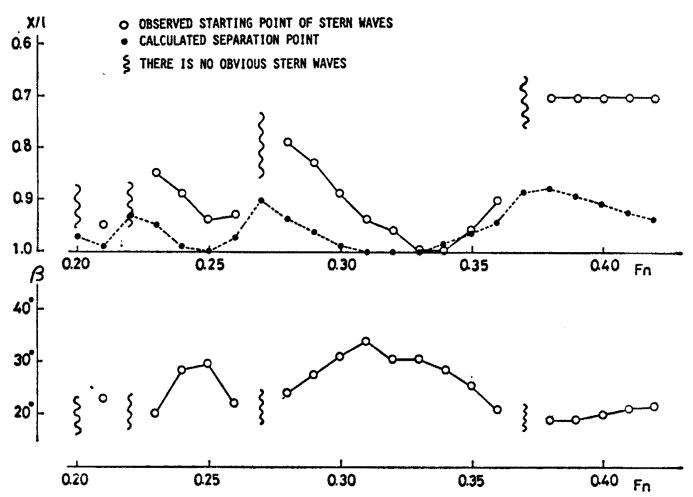

Fig. 4 Starting points of stern waves and angles of stern wave front lines

starting point of stern waves $\left(X_{s w}\right)$ and the angle of their front lines to the $x$-axis $(\beta)$ are shown in Fig. 4.

Towing test and wave analysis were carried out in order to know the relation between formation of stern waves and wave resistance. The model was towed with its trim restrained making use of a parallel guide. Wave resistance coefficient $\left(C_{w}\right)$ was derived with the Schoenherr friction line and form factor $K$ of 0.221 . Wave pattern resistance coefficient $\left(C_{w p p}\right)$ was obtained from the longitudinal wave records on the line of $y / l=1.73$ (at the wall of towing tank) by the Newman-Sharma's method. Both are nondimensionalized by $L^{2}$. The results are shown in 


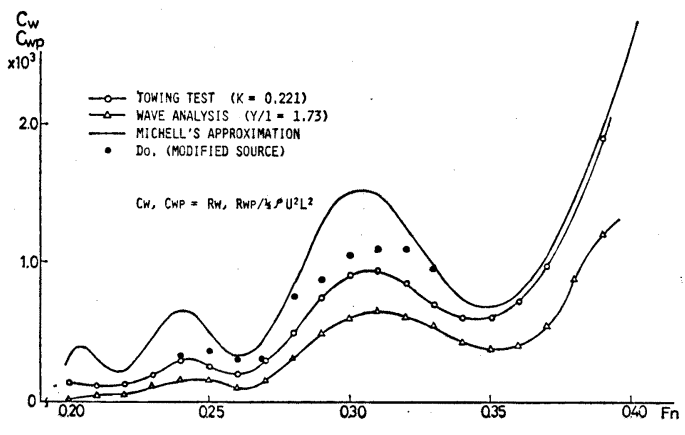

Fig. 5 Wave resistance curves

Fig. 5 together with calculation by the Michell's approximation.

Figs. 4 and 5 show that the starting point of stern waves moves rearward in the velocity range where $C_{w}$ or $C_{w p}$ curves are humps. The stern waves almost disappear where $C_{w}$ curve starts to rise (immediately after the bottom of hollows), for example, at $F_{n}=0.27$ and 0.37 . This relation is reasonably interpreted by considering the phase of bow waves.

The difference between $C_{w}$ and $C_{w p}$ increases in the velocity range of humps and decreases in the range of hollows. This tendency seems to have connection with the intensity of nonlinear stern waves (FSSWs), since nonlinear waves are intensified in the range where $C_{w}$ shows hump (Fig. 2). The variation of $\beta$ in Fig. 4 accords with that of $C_{w}$ curve, which is understood by the linear relation between the intensity of FSSW and $\beta^{4}$ ). It has been pointed out that the resistance due to FSSWs occupies most part of the difference of $C_{w}-C_{w p}{ }^{8}$. Thus, the larger difference between $C_{w}$ and $C_{w p}$ at humps of $C_{w}$ curve is considered due to FSSWs from the stern.

Weighted amplitude functions $A^{*}(\theta)$ derived from wave analysis are shown in Fig. 6 compared with those calculated by the Michell's approximation. The relation between $C_{w p}$ and $A^{*}(\theta)$ is as follows.

$$
C_{w p}=2 \pi \int_{0}^{\pi / 2}\left[A^{*}(\theta) / L\right]^{2} d \theta
$$

At $F_{n}=0.27$ the distribution of $A^{*}(\theta)$ is monotonous which indicates that no interference of linear waves from the bow and the stern occurs. The chain-line at $F_{n}=0.27$ is calculated by the Michell's approximation for the forebody, i.e., the source distribution for the afterbody is ignored. The qualitative accordance of the chainline with the measured is noticeable. These verify the fact that stern waves disappear and only bow waves exist at $F_{n}=0.27$, as is visualized
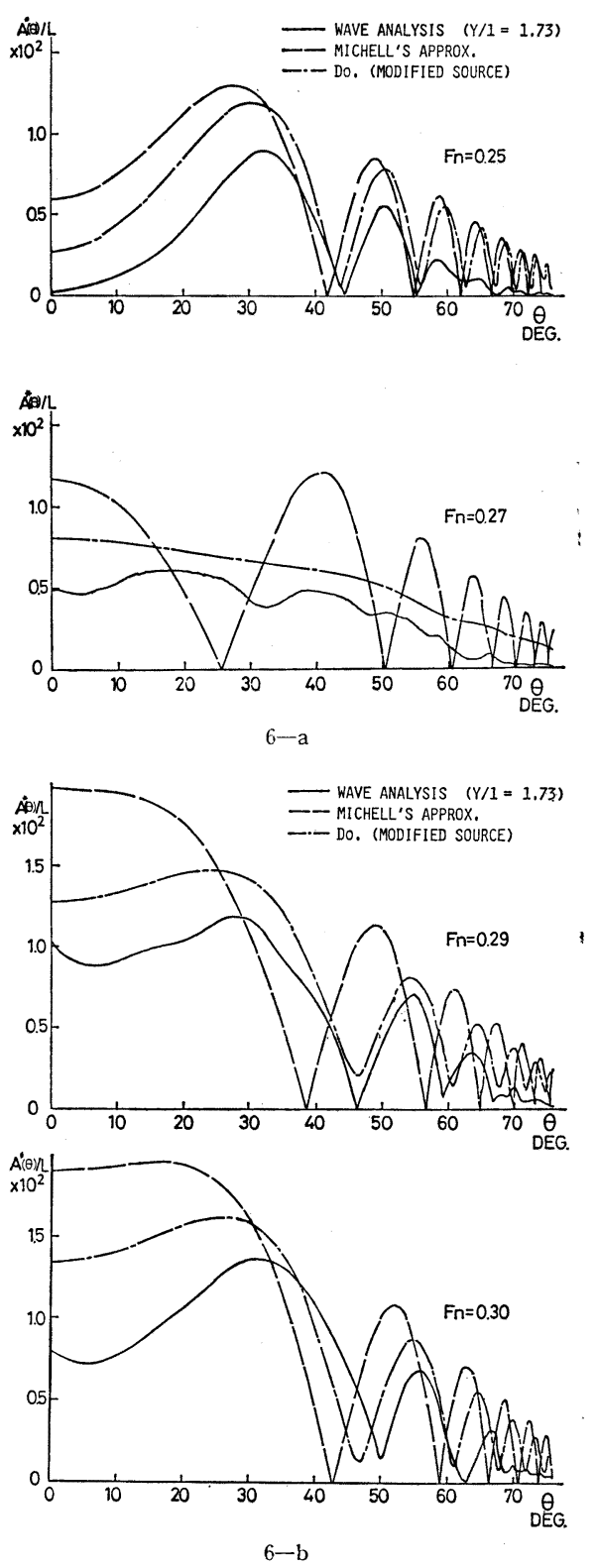

Fig. 6 Weighted amplitude functions

in Fig. 2. As the Froude number increases or decreases from 0.27 , the effect of stern waves appears with their starting points moving. However, the discrepancies between experiment and calculation are obvious.

It should be noted that the movement of the starting point and the attenuation of stern waves play a significant role in the formation of linear waves which dispersively propagate to the farfield.

\subsection{Calculation of separation point}

When a crest of bow wave approaches AP, 
a pressure gradient becomes large at $\mathrm{AP}$, and consequently the point of boundary layer separation moves forward. This effect of waves on boundary layer separation seems to explain the movement of the starting point of stern waves, since stern waves are under the influence of boundary layer.

A 2-D turbulent boundary layer calculation, i.e., momentum integral method (entrainment method), is employed to estimate the position of boundary layer separation. 1/n-th power law, Ludwieg-Tillman's equation and Tompson's chart are made use of for the velocity distribution, the local skin friction and the entrainment function, respectively. The velocity on the edge of boundary layer $U_{e}$ is assumed as,

$$
\begin{aligned}
U_{e}{ }^{2}(x, y, z)= & \left\{U_{f}(x, 0, z)+U_{d}(x, y, z)\right\}^{2} \\
& +V_{d}(x, y, z)^{2}
\end{aligned}
$$

The location $(x, y, z)$ is on the hull surface and $U_{f}(x, 0, z)$ is wavy velocity component in $x$ direction calculated from free wave velocity potential by the Michell's approximation. $U_{d}$ and $V_{\boldsymbol{d}}$ are velocity components of double model flow in $x$ and $y$ directions on the hull surface, respectively. Shape factor is adopted for the estimation of boundary layer separation, and it is assumed that separation occurs when the shape factor becomes 1.8 .

The calculated separation points are shown in Fig. 4. The movement of the separation point qualitatively agrees with that of the starting point of stern waves. It is supposed that bow waves have significant influence on both the generation of nonlinear stern waves and the boundary layer separation and that the wave generation has some connection with the separation.

\section{Evaluation of linear waves by approximation}

\subsection{Modified Michell's approximation}

It is recognized that the shift of the starting point of stern waves remarkably affects the linear wave system generated by the model ship. To exemplify this effect an approximate calculation are carried out by modifying the Michell's approximation.

The source strength given by the Michell's approximation is distributed on the centerplane from FP to the $x$-coordinate where the front line of the foremost stern wave intersect the centerline of the ship model. The source strength from this point to AP is superseded by zero. A similar calculation was attempted before by Suzuki ${ }^{8)}$ with the source distribution independent on $F_{n}$.

\subsection{Results}

The calculated amplitude functions are shown in Fig. 6 in chain-lines. The present calculation is, in general, a better approximation than the original Michell's approximation, reducing the discrepancy between theory and experiment. This indicates that a part of the discrepancy is attributed to the shift of stern waves.

The calculated wave resistance coefficient is shown in Fig. 5. It must be noted that the calculated value at $F_{n}=0.27$ is obtained by the assumption described in 3.2. The wave resistance curve by the modified Michell's approximation approaches to the experimental curve with great attenuation around humps and comparatively small attenuation around hollows. The present approximation can considerably explain the effects of shift and attenuation of stern waves on the wave resistance due to the linear dispersive wave system.

To exemplify the effect of the range of source distribution in the modified Michell's approximation weighted amplitude function is calculated at $F_{n}=0.30$ changing the coordinate of the after end of source distribution. In the range of $1.25 \times N \%$ of ship length forward from AP the source strength is considered zero, i.e., in case $N=0$ source is distributed over the full length of the model and in case $N=10$ over $87.5 \%$ of the model length from FP. The calculated results are shown in Fig. 7, in which eleven calculated results with $N$ from 0 to 10 are compared with the experimental result. With the enlargement of the range where source is not distributed the amplitude function tends to coincide with the experimental one. Although the observed starting point of stern waves approximately coincides with the case of $N=5$, the agreement in amplitude and phase is excellent when $N=10$. At $F_{n}=0.30$ the intensity and the angle of wave front line $\beta$ are so large, as seen in Figs. 2 and 4,

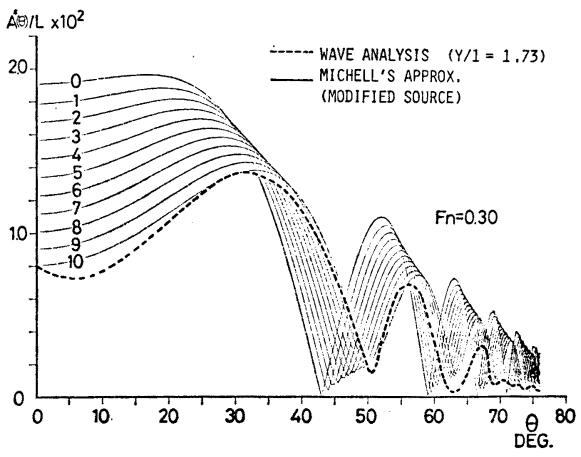

Fig. 7 Weighted amplitude functions calculated by modified source distributions 


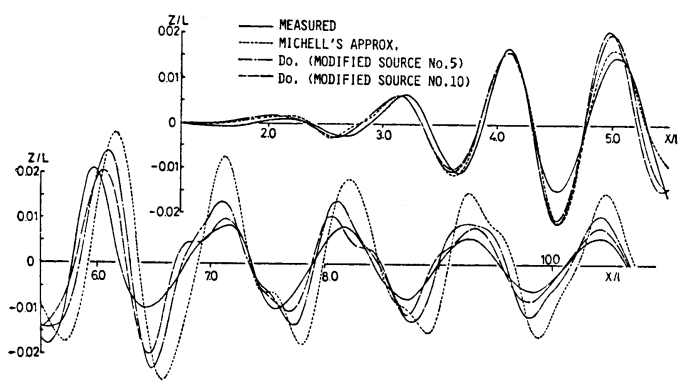

Fig. 8 Longitudinal wave profiles $(y / l=$ $\left.1.73, F_{n}=0.30\right)$

that the displacement effect by the intense nonlinear waves at the stern seems to shift the linear waves from the stern outward. Consequently the virtual starting point of the linear waves is considered to advance forward. Comparison of longitudinal wave profiles on the line of $y / l=$ 1.73 is shown in Fig. 8, in which two profiles by the modified source distributions of the cases of $N=5$ and 10 (denoted No. 5 and No. 10, respectively) are present together with one by the original Michell's approximation. The profile of the case of $N=10$ agrees well with the measured one. The effects of the shift and the attenuation of linear waves from the stern are remarkable, and cannot be ignored for the comprehensive understanding of linear wave resistance. These effects are mostly due to the nonlinear phenomena in the neighborhood of the ship, especially the occurrence of nonlinear waves plays a significant role. It is pointed out that the occurrence of FSSWs gives displacement effect and attenuation effect on linear waves from the bow ${ }^{10)}$. In case the model ship is not so fine as the present model these effects will influence on the two wave systems from the bow and the stern.

\section{Characteristics of free surface shock waves from stern}

\subsection{Description of experiments}

In order to clarify the characteristics of the nonlinear stern waves in the neighborhood of the stern, measurements of wave height and velocity distribution were undertaken at $F_{n}=0.30$. A wave recorder of contact type and a five-hole pitot tube of NPL-type whose outer and inner diameters were $2.1 \mathrm{~mm}$ and $0.4 \mathrm{~mm}$, respectively, were made use of. Wave profiles were recorded on three longitudinal lines at $y / l=0.10,0.15$ and 0.20 and one transverse line at $x / l=1.15$. For the measurement of velocity components two lines were selected, i.e., a line parallel to the centerline at $y / l=0.15$ and a line parallel to the beam at $x / l=1.15$. The five-hole pitot tube was located at $5 \mathrm{~mm}$ to $50 \mathrm{~mm}$ below the disturbed free surface.

\subsection{Experimental results and discussions}

Measured longitudinal wave profiles are shown in Fig. 9. The nonlinear wave from the stern lies on the linear transverse wave from the bow and generates a line of discontinuity at the wave front which are also observed in Fig. 2. Behind the wave front the free surface is unsteady and intense fluctuation is recorded.

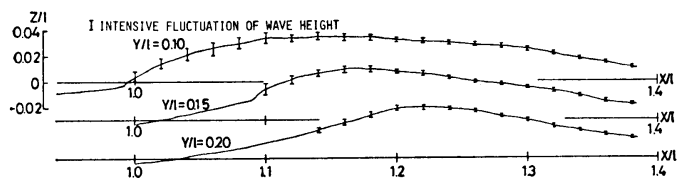

Fig. 9 Longitudinal wave profiles $\left(F_{n}=0.30\right)$

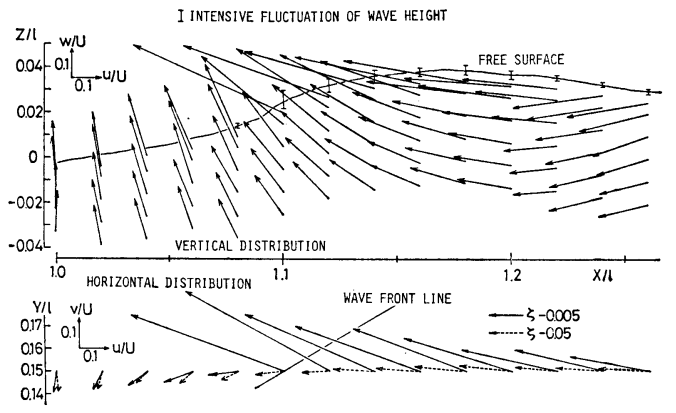

Fig. 10 Disturbance velocity vectors on $x-z$ plane at $y / l=0.15\left(F_{n}=0.30\right)$
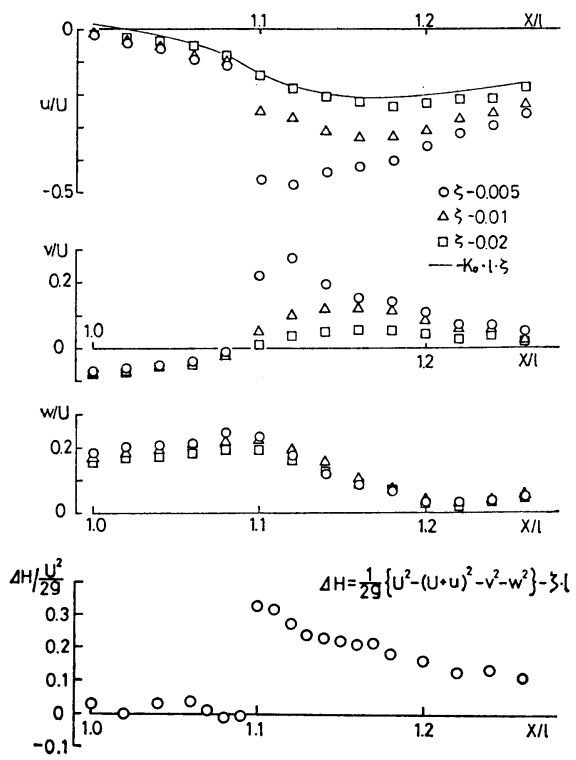

Fig. 11 Disturbance velocity distributions and head loss near the front of nonlinear stern waves $\left(y / l=0.15, F_{n}=\right.$ $0.30)$ 


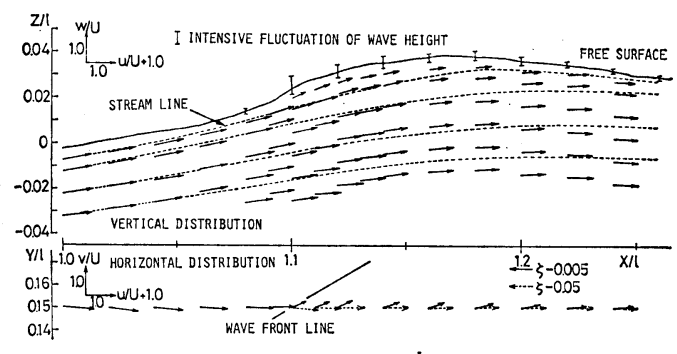

Fig. 12 Velocity vectors on $x-z$ plane at $y / l=0.15\left(F_{n}=0.30\right)$
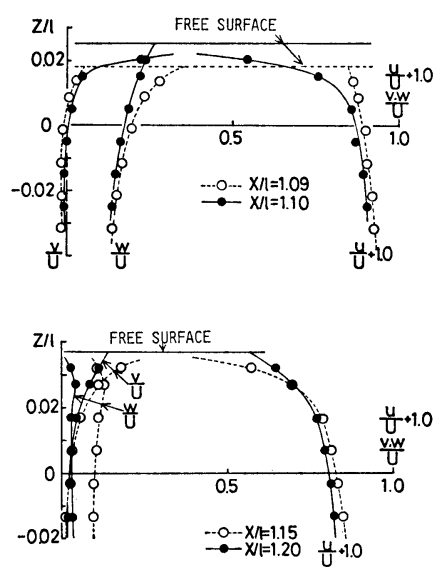

Fig. 13 Velocity distributions near the front of nonlinear stern waves $(y / l=0.15$, $F_{n}=0.30$ )

Results of velocity measurements are shown in Figs. 10 through 15. Disturbance velocity distributions are present in Figs. 10 and 11 which shows great disturbance velocity vectors directed forward and outward behind the wave front in the thin layer near the free surface. The disturbance velocity abruptly increases at the wave front and keeps a great value behind the wave front, though it is gradually attenuated. Fig. 11 clearly shows the discontinuity in velocity, in which the solid line is calculated from the wave height according to the linearized dynamic condition on the free surface, i.e., $u=K_{0} l \cdot \zeta\left(K_{0}=g / U^{2}, \zeta\right.$ is wave height nondimensionalized by $l$ ). The linearized dynamic free surface condition is violated behind the wave front whereas it is approximately satisfied before the front. The head loss is calculated with the measured velocities on the plane $5 \mathrm{~mm}$ below the free surface and measured wave height as shown in Fig. 11. It is evident that head loss is caused by the nonlinear wave phenomenon, which is very similar to turbulent bore. This

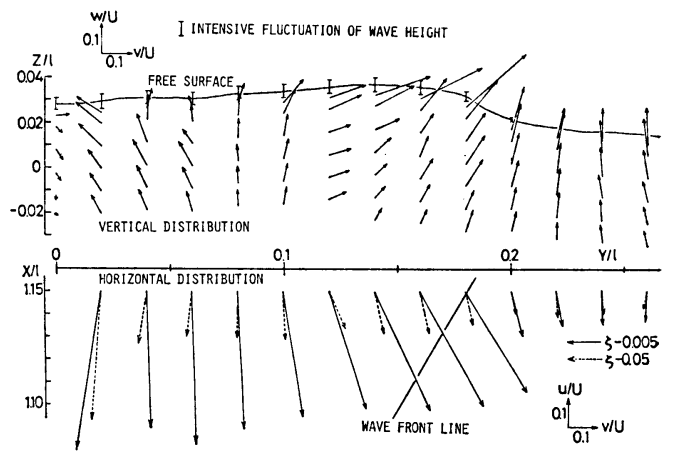

Fig. 14 Disturbance velocity vectors on $y-z$ plane at $x / l=1.15\left(F_{n}=0.30\right)$

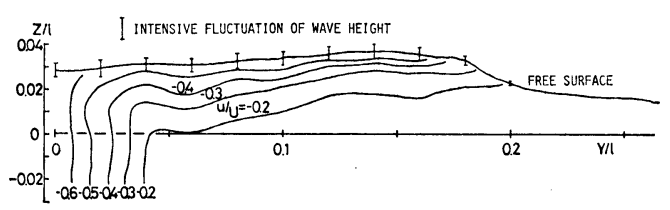

Fig. 15 Contour of disturbance velocity on $y-z$ plane at $x / l=1.15\left(F_{n}=0.30\right)$

head loss will be much greater if velocity components exactly on the free surface were available.

The velocity vectors including uniform flow velocity and the stream lines calculated by these vectors on the same plane of Fig. 10 are shown in Fig. 12. The width between the free surface and the uppermost streamline is considerably increased at the wave front where the absolute velocity suddenly decreases and pressure jump is formed. The lower figure in Fig. 12 indicates that the water behind the wave front moves along the wave front. This, as a result, interrupts the paths of aluminum powder as seen in Fig. 2. Fig. 13 shows depth-wise distributions of measured velocity components. The indication of this figure is that the discontinuity caused by the nonlinear waves produces steep depthwise gradient of velocity and that this slope is attenuated through a kind of diffusive process behind the wave front.

Fig. 14 shows velocity vector distributions on the plane vertical to $x$-axis and Fig. 15 contours of $u / U$ on the same plane. Constantly great disturbance velocities directed forward and outward are evident behind the wave front. The decelerated region in Fig. 15 is in the flow field near the free surface behind the wave front and it is clearly distinguished from the outer region. The wave profile in Fig. 15 is qualitatively common to nonlinear stern waves ${ }^{2}$. The deceleration is not due to the viscous wake but to the 
nonlinear wave motion and it results in the momentum loss far behind.

The precise measurement of velocity component shows the detailed structure of the nonlinear waves from the stern. The properties of the nonlinear waves above-described are essentially same with those of FSSWs around bow. FSSWs occur at any part in the neighborhood of ships depending on conditions.

\section{Fluid motion in the boundary layer}

\subsection{Description of experiments}

FSSWs around sterns have some characteristics different from those around bows. One of them is that they probably interact with the boundary layer. The starting point of FSSWS around sterns varies in the qualitatively same manner with the variation of calculated separation point as described in 3.3.

In order to clarify the relation between boundary layer and free surface waves measurement of velocity components and flow visualization on the hull surface were undertaken. The five-hole pitot tube described in 5.1 and twin-tufts were made use of. Two tufts whose length was $20 \mathrm{~mm}$ were attached on beads which were pierced by a pin so as to be free to circle, and the pin was stuck on the hull surface. One tuft was on the hull surface (inner tuft) and the other was $25 \mathrm{~mm}$ apart from it. The position of the tufts was kept by other fixed beads as seen in Fig. 17. The number of the twin-tufts was limited in order not to disturb the fluid flow too much.

\subsection{Experimental results and discussions}

The measured disturbance velocity distributions are present in Figs. 16-a to c. Distribution of the three velocity components and transverse wave profile are shown in each figures. The velocities in the boundary layer on the plane at $x / l=0.95$ at $F_{n}=0.30$ in Fig. 16-a show that flow is more accelerated at $z / l=-0.02$ than at $z / l=-0.20$ due to the orbital motion of the linear bow waves. The velocity distributions calculated by the 2-D turbulent boundary layer theory described in 3.3 agree with the measurement in spite of some drawbacks of the approximate theory. However, in the thin layer near the free surface the velocity is remarkably decelerated and measurement was impossible at some points on $z / l=\zeta-0.005$ because of the too strong disturbance. The effect of linear wave motion on the velocity distribution in the boundary layer at $x / l=0.85$ at $F_{n}=0.27$ in Fig. $16-\mathrm{b}$ is opposite to the case of Fig. 16-a owing to the change of the phase of linear bow waves, which also agrees with the calculation. Much more large deceleration in the region adjacent to the free surface and turbulence also appear in Fig.
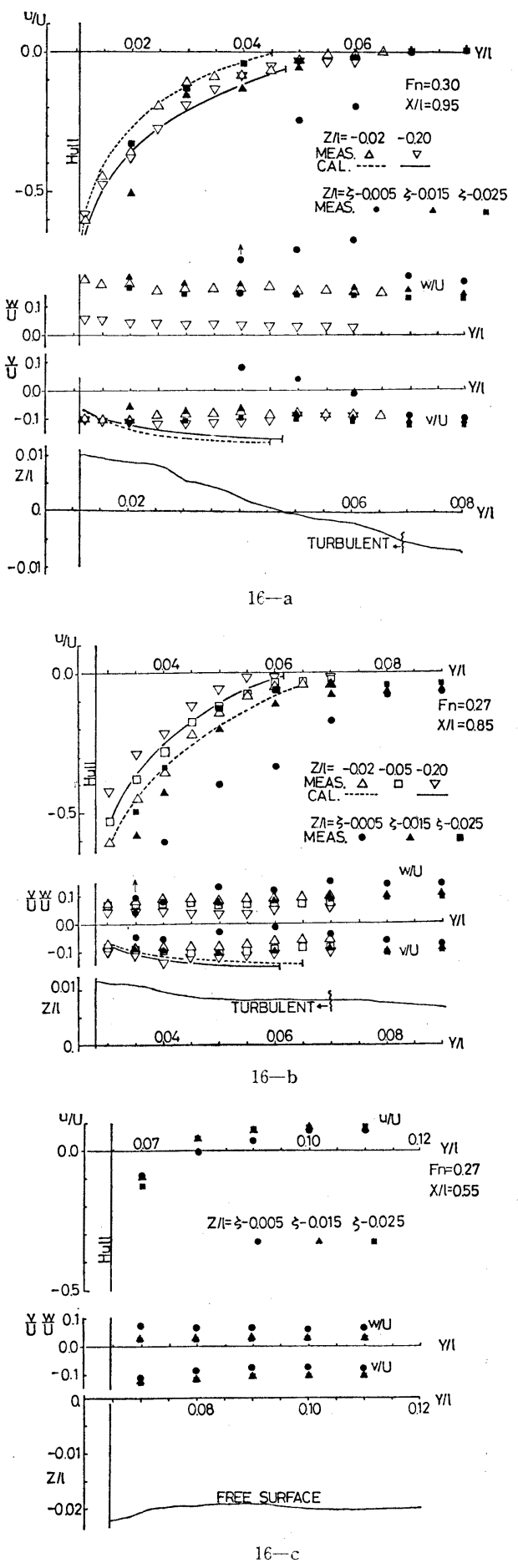

Fig. 16 Disturbance velocity distributions in boundary layer

16-b, but they do not in Fig. 16-c which shows velocity distribution on the plane where nonlinear waves do not exist. This great velocity 


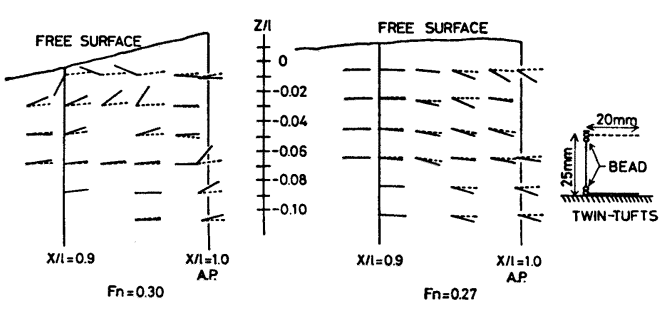

Fig. 17 Observed flow patterns by means of twin-tufts

drop is considered due to the nonlinear motion of FSSWs around sterns.

The visualized flow by twin-tufts which was photographed is shown in Fig. 17. The inner tufts (in solid line) show recirculating motion beneath the stern wave front at $F_{n}=0.30$, while the outer tufts (in dotted line) are all directed aftward. At $F_{n}=0.27$ when FSSW is ultimately weak such a recirculating motion cannot be observed, though the region of 2-D flow separation is supposed to be larger than at $F_{n}=0.30$ as seen in Fig. 4 above. It is supposed that this recirculating flow motion in the boundary layer is mainly caused by the nonlinear violent motion of the FSSWs.

When the number of twin-tufts are increased the starting point of FSSWs and the recirculating flow are shifted forward at $F_{n}=0.30$, which will be owing to the flow deceleration in the boundary layer. The sensitive change of FSSWs around bow due to disturbances is reported by Kayo ${ }^{11}$. FSSWs around sterns are clearly influenced by the linear waves from bows and they interact with boundary layer.

\section{Conclusion}

Principal conclusions are as follows.

1) The stern waves are composed of linear and nonlinear waves, and the latter gives influence on the former.

2) The nonlinear waves around the stern essentially have the same characteristics with free surface shock waves, although they are different in some respects concerned with the interaction with linear bow-waves and boundary layer.

3) The movement of the starting point of stern waves is in close relation with the phase of bow-waves.

4) Flow velocities in the boundary layer is strongly decelerated in the region adjacent to the free surface and a recirculating motion is observed, which are attributed to the motion of the nonlinear waves.

5) The modified Michell's approximation that takes the effects of moving starting point and wave attenuation into account can reduce the discrepancy between linear wave making theory and wave analysis.

\section{Acknowledgement}

This research is partially supported by the Grant-in-Aid for Co-operative Research of the Ministry of Education, Science and Culture. Some parts of experiments were carried out by Mr. T. S. Chong and Mr. M. Takai as a part of their graduation thesis. The authors express their appreciation to them and other colleagues at the Experimental Tank of the University of Tokyo.

\section{References}

1) Y. J. Lin, H. Kajitani, H. Miyata, et. al: On the optimization of the Aft-Part of Fine Hull Forms (First Report), (Second Report), (Third Report), J. of the Kansai Soc. of Naval Arch., Japan, Vol. 177, 179 (1980), Vol. 181 (1981).

2) H. Miyata, Y. Tsuchiya, T. Inui, $H$. Adachi: Resistance Reduction by SternEnd-Bulb (First Report), (Second Report), J. of the Soc. of Naval Arch. of Japan, Vol. 148 (1980), Vol. 149 (1981).

3) H. Miyata, T. Inui, H. Kajitani: Free Surface Shock Waves and Their Effects on Ship Resistance, J. of the Soc. of Naval Arch. of Japan, Vol. 147 (1980).

4) H. Miyata, A. Suzuki, H. Kajitani: Numerical Explanation of Nonlinear Nondispersive Waves around Bow, 3rd Intern. Conf. on Numerical Ship Hydrodynamics, (1981) (to appear).

5) M. Takai, T. S. Chong: Characteristics of Stern Waves and Efficiency of Stern-EndBulb, Graduation Thesis of the University of Tokyo (1981).

6) T. Inui, Y. Kikuchi, T. Iwata: Wave Profile Measurements along Some Mathematical Models by Benzoicacid Film Method, J. of the Soc. of Naval Arch. of Japan, Vol. 100 (1956).

7) T. Inui, K. Masunaga, T. Miura: Photographic Observations of Wave-Making Characteristics of After Hull Bodies, J. of the Soc. of Naval Arch. of Japan, Vol. 101 (1957).

8) N. Kawamura, H. Kajitani, H. Miyata, Y. Tsuchiya: Experimental Investigation on the Resistance Component Due to Free Surface Shock Waves on Series Ship Models, J. of the Kanasi Soc. of Naval Arch., Japan, Vol. 179 (1980).

9) K. Suzuki: Effects of Wake and Wave- 
Breaking on Wave-Making Resistance, Proc. Intern. Seminar on Wave Resistance, the Soc. of Naval Arch. of Japan (1976).

10) H. Miyata: Characteristics of Nonlinear Waves in the Near-Field of Ships and Their Effects on Resistance, Proc. 13th Symposium on Naval Hydrodynamics (1980).
11) Y. Kayo, K. Takekuma: On the Free Surface Shear Flow related to Bow WaveBreaking of Full Ship Models, J. of the Soc. of Naval Arch. of Japan, Vol. 149 (1981).

5), 6), 7) are written in Japanese 